\title{
Disputas en la construcción del Trabajo Social reconceptualizado en la Universidad Católica de Valparaíso (1966-1973)
}

Disputas na construção do Serviço Social reconceitualizado na Universidade Católica de Valparaíso (1966-1973)

Disputes in the construction of reconceptualized Social Work at the Catholic University of Valparaíso (1966-1973)

\section{Leticia Arancibia Martínez*}

\begin{abstract}
Resumen - A partir de la investigación sobre el movimiento de reconceptualización del Trabajo social, en la Escuela de Trabajo social de la Universidad Católica de Valparaíso, se analizan las condicionantes y la articulación de diferentes tiempos y movimientos, locales y globales, del proceso de los 60 hasta 1973, que dieron forma al Proyecto de Escuela. Este expresa la producción y circulación del conocimiento sobre el problema de lo social en América Latina, la recepción de corrientes marxistas y la crítica hacia una formación universitaria ajena de la sociedad, conservadora ante el proceso político de la realidad chilena. Cuestiona el desarrollismo y la dependencia económica y cultural de los países del Sur, en el estado capitalista, asumiendo demandas de transformación. En el escenario de la vía chilena al socialismo, diferentes lógicas se disputaron y articularon, movilizando discusiones políticas, teóricas y metodológicas, profundizando y materializando un proyecto de conciencia crítica del sentido y orientación del Trabajo social.

Palabras-clave: reconceptualización del trabajo social; imaginarios sociales; procesos políticos; producción del conocimiento; Valparaíso.
\end{abstract}

Resumo - A partir da pesquisa sobre o movimento de reconceituação do Serviço Social, na Escola de Serviço Social da Universidade Católica de Valparaíso, analisam-se os condicionantes e a articulação de diferentes tempos e movimentos, locais e globais, do processo dos anos 60 até 1973, os quais deram forma ao Projeto da Escola. Este expressa a produção e circulação de conhecimento sobre o problema do social na América Latina, a recepção das correntes marxistas e a crítica a uma formação

\footnotetext{
* Doctora en Sociología por la Université Catholique de Louvain. Profesora de la Escuela de Trabajo Social de la Pontificia Universidad Católica de Valparaíso. Correspondência: Pontificia Universidad Católica de Vaparaíso Escuela de Trabajo Social: Edificio Monseñor Gimpert. Avenida Brasil 2830, Valparaíso, Chile. Email: <leticia.aran cibia@gmail.com>.
} 
universitária alheia à sociedade, conservadora do processo político da realidade chilena. Questiona também o desenvolvimentismo e a dependência econômica e cultural dos países do Sul no estado capitalista, e assume as demandas de transformação da época. No cenário da chamada "via chilena ao socialismo", diferentes lógicas se disputaram e articularam, mobilizando discussões políticas, teóricas e metodológicas, aprofundando e materializando um projeto de consciência crítica sobre o sentido e orientação do Trabalho Social.

Palavras-chave: reconceituação do trabalho social; imaginários sociais; processos políticos; produção de conhecimento; Valparaíso.

\begin{abstract}
From research on the reconceptualization movement of social work at the School of Social Work at the Pontifical Catholic University of Valparaíso, this article analyzes the conditions and the articulation of different times, and both local and global movements, from the 1960s to 1973, which gave shape to the project of the school. This project proclaims the production and circulation of knowledge about social problems in Latin America, the reception of Marxist currents, and the criticism of university education apart from society, conservative of the political process of the Chilean scenario. It also questions developmentalism and economic and cultural dependence of countries of the South in the capitalist state, and takes on the demands for transformation of the time. In the scenario of the so-called "Chilean way to socialism," different logics disputed with each other and were articulated, mobilizing political, theoretical and methodological discussions, deepening and materializing a project of critical awareness about the meaning and orientation of social work.
\end{abstract}

Keywords: reconceptualization of social work; social imaginary; politics processes; production of knowledge; Valparaiso.

\title{
Introducción
}

La reconceptualización del Trabajo social es un movimiento que se extendió por la mayoría de los países latinoamericanos, entre las décadas de los 60 y 80, con temporalidad y efectos diferentes, marcado por las fluctuaciones, crisis y acuerdos de cada contexto nacional. En él, se destaca el proceso de articulación que se dio entre saberes, tendencias y escuelas en las discusiones y los cambios en la formación a nivel nacional y latinoamericano.

En el análisis de las discusiones, los problemas, las interpretaciones, las matrices teóricas y los imaginarios sociales sobre el problema de lo social y el Trabajo social en América Latina, el escenario donde despliega las "tendencias de análisis e interpretaciones acerca de su propia intervención y sobre la realidad social" (YAZBEK, 2009, p. 2) está en interlocución con "el propio movimiento de la sociedad". Los procesos de cambio en las disciplinas "no son un producto meramente endógeno de cada profesión. Se generan y se articulan con la dinámica social específica que se registra en un momento histórico determinado" (ALAYÓN, 2007, p. 9).

Así, los conocimientos que se producen y que circulan en el campo teórico y sus expresiones en las relaciones sociales, las significaciones sobre 


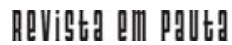

\} DISPUTAS EN LA CONSTRUCCIÓN DEL TRABAJO SOCIAL - MARTÍNEZ, L. A. \}

DOI: $10.12957 /$ REP.2017.32721

el problema de lo social y la profesión, durante la reconceptualización, se explica a través de la dinámica social y las posiciones en el contexto social, político y económico de las sociedades capitalistas (IAMAMOTO, 2003) durante el período entre los 60 y los 80 . Así como las perspectivas y las "visiones de mundo" (COUTINHO, 1991), que "implican [...] no solo una representación de aquello que es, sino también, y sobre todo, una representación de aquello que debe ser, una visión de mundo de lo real con normas de acción adecuadas" (COUTINHO, 1991, p. 10), con elementos instituidos e instituyentes (CASTORIADIS, 1975, 2004) en lo social.

Bajo esta consideración, analizaremos el proyecto "La producción y circulación del conocimiento en la formación universitaria sobre el problema de lo social en América Latina en las décadas 60 y 70: una lectura comparada de la investigación y la enseñanza del Trabajo Social en Chile y Brasil", DII № 039.328/2016, financiada por la Pontificia Universidad Católica de Valparaíso, que sirve de base para este artículo, es resultado de la investigación dirigida por Leticia Arancibia Martínez, profesora de la Escuela de Trabajo social de la Pontificia Universidad Católica de Valparaíso (PUCV), junto a los co-investigadores Gloria Cáceres Julio, Carlos Valdebenito V. y Pamela Soto García integrantes del Núcleo de Imaginarios sociales, Memoria y Política de Valparaíso, de la Escuela de Trabajo social de la PUCV. Esta investigación está asociada con la pesquisa "O Movimento de Reconceituação do Serviço Social na América Latina: determinantes históricos, interlocuções internacionais e memória", financiada por $\mathrm{CNPq}$ Processo [421744/2016-2] - Universal 01/2016 - Faixa C - até R\$120.000,00 - cuya Pesquisadora responsable es la profesora Marilda Villela lamamoto.

Para analizar las relaciones entre la formación universitaria y las perspectivas profesionales a partir de la reconceptualización, distinguiremos los elementos principales del contexto sociopolítico chileno y latinoamericano durante la reconceptualización en los años 60 y 70; analizaremos las articulaciones de las discusiones y lecturas sobre el problema de lo social en Chile, distinguiendo los imaginarios sociales expresados en el proceso histórico del gobierno de Salvador Allende y la experiencia de la vía chilena al socialismo de la Unidad Popular, donde se ponen en tensión un Trabajo social crítico y uno conservador, así como las disputas entre los grupos que impulsaban los cambios, respecto del énfasis y prioridades en la marcha de las transformaciones políticas, económicas, sociales y culturales del momento.

\section{El problema de lo social y el contexto de la reconceptualización en América Latina}

Entendiendo el Trabajo social como "un producto histórico [...], como tal, adquiere sentido e inteligibilidad en la historia de la sociedad de 


\section{ReVistg all paltg}

\} DISPUTAS EN LA CONSTRUCCIÓN DEL TRABAJO SOCIAL - MARTÍNEZ, L. A. \}

DOI: $10.12957 /$ REP.2017.32721

la que es parte y expresión" (IAMAMOTO, 2003, p. 221), el análisis de los procesos de formación, producción y circulación de conocimientos, no pueden estar ajenas a esa inscripción social e histórica. La dialéctica entre lo material y los sistemas de representaciones que se producen en las relaciones sociales, en lo histórico-social, donde los individuos se representan el mundo y pueden llegar a desarrollar conciencia de las contradicciones, nos ubican en el reconocimiento de la ideología, y la crítica al idealismo, donde "el factor que en última instancia determina la historia es la producción y la reproducción de la vida real" (ENGELS apud MARX; ENGELS, 1974). El estudio de la conciencia se encontrará siempre con la ideología.

no creen que puedan eliminar mediante el 'pensamiento puro' a sus amos industriales y su propia humillación práctica. Se dan cuenta muy dolorosamente de la diferencia que existe entre el ser y el pensar, entre la conciencia y la vida. Saben que la propiedad, el capital, el dinero, el trabajo asalariado, etc., no son precisamente quimeras ideales de sus cerebros, sino creaciones muy prácticas y muy materiales de su auto enajenación, que solo podrán ser superadas, asimismo, de un modo práctico y material, para que el hombre se convierta en hombre no solo en el pensamiento, en la conciencia, sino en el ser real, en la vida. (MARX; ENGELS, 1967, p. 118).

Analizar el cambio en las representaciones, exige considerarlas no como mero reflejo de las formas de relación, sino como la expresión que se entabla por el conflicto, en las relaciones sociales. El conflicto entre objetivaciones y explicaciones de las relaciones sociales de producción, que operan como ideología. Aquí recurrimos a los imaginarios sociales (CASTORIADIS, 1975, p. 225) que "designan la colectividad no como simple definición o función taxonómica, sino, al mismo tiempo, como comprensión y cualidad o propiedad, de la sociedad que se instituye en esa operación. Estas imágenes están en una relación dialéctica con las necesidades construidas en cada sociedad específica". Considerando la operación de mecanismos socio-históricos y psíquicos en la construcción de los imaginarios, dicha imagen del mundo, permite la justificación y la reproducción de las relaciones sociales, pero al mismo tiempo expresa el conflicto, y produce nuevas significaciones. Esta perspectiva cuestionará el proceso ciego de institución de la sociedad, en el que se ocultan las condiciones, impidiendo que los sujetos sean capaces de reconocerse desde su participación activa de la construcción social (CASTORIADIS, 2004) y la apropiación del mundo, desde las condiciones materiales y simbólicas de la acción humana.

Los hombres son los productores de sus representaciones, de sus ideas, etc., pero los hombres son reales y actuantes, tal y como se hallan condicionados por un determinado desarrollo de sus fuerzas productivas y por el intercambio que a él corresponde, hasta llegar a sus formaciones más amplias. La conciencia no puede ser nunca otra cosa que el ser 
consciente, y el ser de los hombres es su proceso de vida real. Y si en toda la ideología los hombres y sus relaciones aparecen invertidos como en la cámara oscura, este fenómeno responde a su proceso histórico de vida, como la inversión de los objetos al proyectarse sobre la retina responde a su proceso de vida directamente físico. (MARX; ENGELS, 2014, p. 27).

Las condiciones históricas en las que se desarrolla el movimiento de reconceptualización del Trabajo social, se corresponden con los procesos sociales y económicos, que Netto ubica dentro del "agotamiento de un patrón de desarrollo capitalista - de las "ondas largas" de crecimiento". (NETTO, 2007, p. 73). Las economías y gobiernos latinoamericanos, sujetos al influjo de las políticas globales y al orden financiero, organizado desde la segunda guerra, con los acuerdos de Bretton Woods, incidirán en el énfasis del desarrollo de las décadas del 50 al 70 (MARTíNEZ; REYES, 2012, p.37). Como parte de esta apuesta, el modelo de sustitución de importaciones en su primer momento 1950-1964 (MARTíNEZ; RANGEL, 2012), con las políticas de la Cepal y la Alianza por el Progreso de Estados Unidos (1962) buscaban el aumento de las fuerzas de producción, y la expansión de la economía en los países subdesarrollados, para generar la demanda de bienes de capital hacia las economías de los países hegemónicos. Se promoverá una participación mayor del estado, la inversión en industrialización, infraestructura, educación y salud (KUCZYNSKI, 2003). Estratégicamente apuntarán hacia la contención del descontento social de diversos actores, cuyo mayor posicionamiento político y elevación de las expectativas y el influjo de la revolución cubana en el imaginario social, marcará un escenario donde la revolución y el cambio permea las representaciones sobre la lucha política y social de movimientos sociales durante ese período, fortaleciendo su organización y articulación a nivel nacional.

El fin unilateral de los acuerdos de Bretton Woods por parte de Estados Unidos en 1971, cristalizará nuevos intereses para reemplazar las políticas de corte keynesiano, dando lugar a una presión progresiva, a los países del sur, a través de la violencia de las dictaduras, para asegurar la expansión del capital. El segundo período, de 70 a 80, desencadenará la crisis de la deuda y otros efectos en las grandes oleadas del capital, que se expresarán progresivamente hasta hoy en América Latina, con el orden neoliberal.

\section{El movimiento de reconceptualización y la articulación entre escuelas de Latinoamérica}

El movimiento de Reconceptualización del Trabajo social es un proceso que se extendió por varios países latinoamericanos, con una temporalidad y efectos diferentes. En el caso del Cono Sur, en la década de los 60 se viven procesos de cambio y cuestionamiento de círculos académicos 


\section{Revistg all paUtg}

\} DISPUTAS EN LA CONSTRUCCIÓN DEL TRABAJO SOCIAL - MARTÍNEZ, L. A. \}

DOI: $10.12957 /$ REP.2017.32721

y profesionales del Trabajo social ante la situación de dependencia de la profesión respecto de las concepciones, métodos y conocimientos presentes en la formación profesional. La reconceptualización surge como producto de la crítica, que se realiza hacia el modo en que se configura el Trabajo social, ante los métodos tradicionales, la ideología implícita y su falta de pertinencia y respuesta a la realidad latinoamericana, enfrentada a las demandas y expectativas de diferentes grupos de la sociedad respecto de los cambios sociopolíticos en la región (DE LA VEGA, 1976; PALMA, 1974).

El desarrollo de encuentros y documentos, serán un espacio de crítica inicial "en el seno de grupos de trabajadores sociales" (KISERMANN, 2007:35) que cuestionan la profesión, realizan "un profundo estudio de la realidad latinoamericana, su subdesarrollo y creciente dependencia económica [...], analizando métodos, técnicas y procedimientos, categorías de análisis de la realidad y su práctica institucional y formativa de trabajadores sociales, y de búsqueda en el sentido de lograr alternativas científicas" (KISERMANN, 1998). Partiendo por un énfasis técnico metodológico, reconocen el nuevo contexto político, los procesos de cambio, los contenidos y las discusiones que se darán en Seminarios y Congresos de Trabajo social en América Latina (Porto Alegre, 1965, Montevideo, 1966, Caracas, 1968, Ambato 1971); se profundizará la crítica desde una mayor presencia de la corriente marxista (Concepción 1969, Cochabamba, 1970, Chillán, 1973), opuesta a la visión instrumental de la profesión, impactada por el positivismo y por las orientaciones de la planificación bajo la matriz desarrollista de la Cepal (Prebisch, Cardoso y Faletto).

lamamoto y Netto reseñan el "papel destacado en la articulación de las inquietudes profesionales del continente" (IAMAMOTO, 2003, p. 228) por parte de Brasil, Argentina, Chile y Uruguay, dado un escenario de abierto cuestionamiento a la crisis del capitalismo. En este proceso, Netto destaca cuatro factores que aportarán el proceso de reconceptualización al Trabajo social: i) la articulación de una nueva concepción de la unidad latinoamericana; ii) la explicitación de la dimensión política de la acción profesional; iii) la interlocución crítica con las Ciencias sociales y iv) la inauguración del pluralismo profesional (NETTO, 2007, p. 77).

El proceso de la reconceptualización del Trabajo social se da en la existencia de una acumulación de tiempos desiguales que se articulan y convergen en un espacio determinado (Santos, 1985). Estos tiempos diferenciados permitirían explicar la fuerza que cobra en América Latina, el impulso o protagonismo de algunas Escuelas y el mutuo influjo de ideas, discusiones y perspectivas. En Chile, se postulará el desafío de contribuir, hacia las transformaciones políticas, sociales y económicas, en crítica a la dependencia del capitalismo, considerando el Trabajo social como actor dentro de ese proceso. En el caso de Brasil (NETTO, 1998), las discusiones y gérmenes de crítica, que se desarrollaban en algunas Escuelas, serán interrumpidas por el golpe de Estado en 1964, construyendo espacios de 
resistencia, pero generando un éxodo de profesores a otros países de la región. En Chile, instituciones estatales y universidades acogerán intelectuales brasileños. Paulo Freire llega asesorando la Reforma agraria y el Ministerio de Educación. En la Escuela de Trabajo social de la Universidad Católica de Valparaíso, Ilegarán Vicente Faleiros y Eva Oliveira.

\section{Los procesos en el Trabajo Social chileno. Circulación de debates y experiencias en el proceso de la reconceptualización}

En las décadas del 60 y 70 la circulación y producción de saberes provenían desde distintas orientaciones. Por una parte, corrientes de inspiración cristiana católica (NETTO, 2007), asociadas a la reforma de la iglesia y a conceptos de liberación, promoción y educación popular, y por otra parte, corrientes de orientación marxista que plantearán conceptos como lucha de clases, independencia y articulación social-popular (IAMAMOTO, 2003; ALAYÓN, 2007; NETTO, 2007). Estas corrientes tendrán diferentes desarrollos en los países, bajo el imaginario de unidad latinoamericana y el intercambio académico entre Escuelas, que permearán ideas, saberes, prácticas e imaginarios sociales generando nuevas y múltiples significaciones imaginarias sociales (CASTORIADIS, 1975, 2004) que participan de la coconstrucción y circulación de conocimientos y prácticas, con diversas expresiones.

En la Escuela de Trabajo social de la Universidad Católica de Valparaíso, se experimentará un proceso de crítica sobre el Trabajo social tradicional (PROYECTO ESCUELA UCV, 1972), en el cual se articularán varios tiempos en el contexto nacional: las características del proceso político y social en curso en Chile; el proceso de Reforma universitaria y Educacional en curso, y la trayectoria del Trabajo social chileno, en medio del cuestionamiento ante el papel del estado capitalista, y un rol paliativo, que no considera las demandas redistributivas, limitándose a asegurar la reproducción de un sistema de explotación y opresión, evidenciada en la miseria, las injusticias y la exclusión experimentada por segmentos completos de personas y grupos de la sociedad.

Como antecedente, el gobierno demócrata cristiano (1964-1970), bajo la consigna de "revolución en libertad" aplicó políticas desarrollistas, sujeto a la influencia de Estados Unidos, con la Alianza por el progreso (Doc. Básicos, 1962); y la democracia cristiana alemana, con la Fundación Konrad Adenauer, que destinaron políticas hacia la región. Disputando la hegemonía en el intento de contener el descontento y hacer frente a movimientos populares, y la amenaza de la extensión de luchas de liberación y anticoloniales, impactadas por la experiencia de la revolución cubana. Se distingue el imaginario revolucionario, en movimientos de izquierda en gran parte de América Latina, cuestionando la dependencia asfixiante y opresiva del capitalismo a escala mundial. 


\section{ReVistg all paltg}

\} DISPUTAS EN LA CONSTRUCCIÓN DEL TRABAJO SOCIAL - MARTÍNEZ, L. A. \}

DOI: $10.12957 /$ REP.2017.32721

Hoy día la América Latina se encuentra claramente en la mitad de una revolución de grandes alcances. No es una revolución comunista, y ni siquiera inspirada por los comunistas. Es una revolución contra la pobreza, el analfabetismo, la injusticia social y la desesperación humana. (Extracto del Discurso de Teodoro Moscoso, 15 de febrero 1962, en Alianza por el progreso, Documentos básicos).

La vocación transformadora de la crítica al capitalismo en Chile y Latinoamérica, irá tomando mayor densidad y presencia, partiendo aguas respecto de las definiciones de la profesión, diferenciará un trabajo social crítico, consciente de su posición en la división del trabajo, el alcance histórico de su origen y desarrollo, y un servicio social tradicional, cuyo quehacer focalizado en un individuo, descontextualizado, se rebelaba incapaz de responder ante la demanda de la profesión. Para Diego Palma, la reconceptualización expresa la permanente disputa entre un Trabajo social crítico y uno de reproducción. Esto se observa en el proceso, en el contexto de encuentros y seminarios, organizados por el grupo ECRO en la Argentina, donde confluirán ideas reformistas, junto a postulados críticos.

Esa fue la primera etapa, [...] duró un par de años y ellos organizaron los primeros seminarios latinoamericanos de trabajo social, encontraron a la gente pero ellos lo que querían era como capturar toda esta gente joven, de ese momento, bajo esta influencia más bien reformista, modernizante si tú quieres. Pero hubo un seminario en Montevideo [...], y ahí se encontró este grupo en que estaba Vicente, estaba la Ana María Quiroga, la Consuelo es posterior, había una niña de Bolivia que se llamaba Teresa Sheriff. (DIEGO PALMA, entrevista 2016).

El énfasis racionalizador de las apuestas de algunas escuelas de Trabajo social en el proceso de cambio de la formación y la práctica profesional, si bien, serán una actualización de las demandas del conocimiento, contienen, no obstante, el intento homologador de los métodos al marco de la racionalización positivista, de mediados de los 60 (NETTO, 2007). Un cierto metodologismo (FALEIROS, 2007) se impone como estrategia de avance profesionalizante del Trabajo social, así como vehículo modernizante, que permitía establecer relaciones entre diferentes posturas políticas alojadas en las escuelas. La politización será determinante para dirimir estas tensiones.

En Chile, la estrecha asociación entre la reconceptualización y los procesos políticos en curso, se verán reforzados en el contexto de la Reforma universitaria que inicia en 1967, en un movimiento que demandará la "democratización de las estructuras docentes y la transformación de los perfiles académicos y curriculares, lo que crea espacios para la reflexión y la emergencia de propuestas renovadoras de la visión teórica y de los métodos de intervención profesional" (RUZ; HERNÁNDEZ, p. 90). La articulación política al interior de la universidad, reforzará los procesos desencade- 
nados por la reforma universitaria, pero al mismo tiempo, tomará el peso de las resistencias de los sectores conservadores del país, ante el nuevo escenario donde el Trabajo social cobrará protagonismo.

\section{Remando al interior de la institucionalidad universitaria de la UCV. Los procesos y tensiones políticas y teóricas, hacia la praxis de liberación del hombre dominado}

El proceso de la reconceptualización del Trabajo social en la Universidad Católica de Valparaíso, se da en la confluencia de distintos procesos, a fines de la década 1960, poniendo al centro una crítica profunda a la situación de dependencia y a la forma en que la Universidad plantea su quehacer, en una dinámica de politización de la sociedad chilena.

Junto al cuestionamiento de la estructura organizativa y formas del poder en la Universidad, y las necesidades concretas impuestas ante el quehacer de los profesionales, el Proyecto de la Escuela de Trabajo Social de la UCV, expresa un auto cuestionamiento desarrollado por alumnos y docentes de las Escuelas de Servicio social y Educación Familiar. Este proceso se inicia en el año 1966 en la Escuela de Educación Familiar, donde se produce una crisis interna, formándose una comisión interna (alumnos, docentes y autoridades académicas) para analizar los problemas de la formación. Posteriormente, en junio de 1967, el cuestionamiento en el Consejo superior y la organización estudiantil, inician el proceso de Reforma Universitaria en la Universidad Católica de Valparaíso, que conducirá a profundas transformaciones en la estructura académica y de poder, como la elección directa de rector y otras autoridades académicas (ALLARD, 2017).

En 1968, la Escuela de Servicio Social inicia un proceso de "auto cuestionamiento de la profesión y de la formación profesional a partir de las exigencias de la Realidad Chilena y Latinoamericana" (PROYECTO, p. 12). En ese contexto, el 11 de octubre de 1968 se realiza la Primera Convención de la Escuela de Servicio Social, donde profesores y estudiantes debatieron sobre dos proyectos de plan de estudios, presentados, el primero, por un grupo de estudiantes y profesores, que proponen la reestructuración de la escuela, bajo "la crítica y la responsabilidad con los cambios y procesos socioculturales que la realidad exige" (Ponencia 1, Convención 1968, p.1). El segundo, de algunos profesores de prácticas, plantea el servicio social como agentes de cambio (Ponencia 2, 1968, p. 2). La mayoría de la comunidad de la escuela, eligió el primero. Luego, en 1969 se forma una comisión de estudiantes y profesores de las Escuelas de Educación Familiar y de Servicio Social, que analizarán ambas profesiones desde lo teórico y lo práctico, proponiendo una nueva forma del quehacer y la creación de una Escuela de Trabajo social. 


\section{ReVistg all paltg}

\} DISPUTAS EN LA CONSTRUCCIÓN DEL TRABAJO SOCIAL - MARTÍNEZ, L. A. \}

DOI: $10.12957 /$ REP.2017.32721

En ese período se desarrollarán cursos de actualización en Ciencias Sociales (Dialéctica y Marxismo, Metodología, Filosofía, Sociología), para estudiantes y profesores de la Escuela, dictados por profesores de diferentes disciplinas del Instituto de Ciencias sociales y desarrollo de la UCV. Dentro de los profesores aportan Vicente de Paula Faleiros, con formación en marxismo, así como el chileno Víctor Farías quien había estudiado Marx en Heidelberg, en lengua original, imprimiendo mayor profundidad en las lecturas, tales como las Tesis de Feuerbach, el Capital, y otros textos, intentando llevar el estudio más allá de los manuales, traducciones y lecturas de bolsillo, que circulaban en la época.

en Valparaíso fue muy importante Farías, fue muy importante porque [...], nosotros de marxismo conocíamos el manifiesto, conocíamos algo de Mao y yo te diría que no mucho más ah, o sea, pa' que te voy a decir que nos habíamos metido a leer El Capital, no. [...], los Grundrisse, eso apareció después, te fijas, no había edición en español de Grundrisse, por eso estábamos muy amarrados conceptualmente, éramos muy limitados conceptualmente, como pa' poder pensar de manera creativa la importancia de este tipo de intervención particular pero concreta [...] Que no es esperar que se produzca la revolución pa' que vengan todos los cambios. (DIEGO PALMA, entrevista 2016).

En el proyecto de la Escuela de la Universidad Católica de Valparaíso, se criticará el rasgo moralizante -teleológico del Trabajo social, que tuvo su impacto en las escuelas chilenas, referido por Ruz y Hernández (2007). En el contexto de la crisis se genera un quiebre a la continuidad de la formación, con el cuestionamiento de la profesión y el auto cuestionamiento por parte de los profesores. La apertura hacia nuevas corrientes y hacia el marxismo, estará dada ante la magnitud del desafío de refundar la escuela, que implicaba derribar las bases conservadoras del Trabajo social, construyendo una escuela reconceptualizada, a la altura de los desafíos de la transición al socialismo.

los profesores estaban como esponja, o sea, a cualquier cosa que uno les pudiera plantear [...], porque qué pasó que, el marxismo, aquí la democracia cristiana era muy fuerte, lo cual quiere decir que mucha gente recibió formación de tipo Vekemanns, por el lado de la teoría de la marginalidad, una cosa cultural pero cultural de derecha, o sea, de esta gente que viaja del campo a la ciudad con sus canastos, con su cultura y que no se adapta, entonces que es culpa de ellos que vivan en la marginalidad porque no se han adaptado a lo que es la cultura urbana moderna, entonces siguen siendo primitivos campesinos que viven en los márgenes de la ciudad. Por la militancia resulta que la formación [...] que la gente recibía como militante, era de frasecitas, citas, consignas. Pero le empieza a decir otra cosa, entonces ¿Cómo nos ponemos al día, cómo nos actualizamos?, o sea, ¿cómo somos capaces de manejar esto? En-tonces vino como un período de reciclaje muy rápido. (DIEGO PALMA, entrevista 30 de Septiembre 2016). 


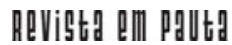

\} DISPUTAS EN LA CONSTRUCCIÓN DEL TRABAJO SOCIAL - MARTÍNEZ, L. A. \}

DOI: $10.12957 /$ REP.2017.32721

El 8 de junio de 1970 se constituye una comisión interescuela de profesores y estudiantes, con estatuto oficial el 21 de octubre de 1970, para elaborar el nuevo Proyecto de la Escuela de Trabajo Social. El 20 de abril de 1971, el proyecto es sometido a la discusión del Senado académico de la Universidad, donde sectores conservadores cuestionarán "la interpretación histórica y la línea general del proyecto" (QUIROZ, 1972, p. 36). Se desarrollará un debate intenso, reflejando las discusiones centrales sobre proyectos de sociedad, y el rol de la Universidad. Así, la relación entre marxismo y cristianismo; "el rol de una Universidad que se dice comprometida con el pueblo que en la práctica no lucha por sus intereses; El marco de análisis que se usaba para interpretar la historia y la realidad actual" (p. 36); el quehacer profesional y "su diferencia con el quehacer político" (p. 36). El Proyecto será aprobado por unanimidad el 27 de junio de 1971, y se organizará a través de: el Análisis de la Realidad Nacional, el Objeto del Trabajo Social; el Quehacer Profesional; los Objetivos del Trabajo Social; los Campos de Accioìn; y la Metodología.

El punto de partida del proyecto es la Realidad nacional, poniendo al Trabajo Social referido a una realidad concreta: el estudio de la realidad chilena en su situación de dependencia de un orden internacional y los desafíos del quehacer profesional.

En el caso de nuestra Escuela se plantea como necesario ubicarse en la Realidad chilena para entender las tareas y desafiìos que dicha realidad presenta, [la cual] se entiende mejor dentro del marco de la dependencia; vale decir, que la realidad social, econoìmica, poliìtica y cultural, se situì al interior del sistema internacional del cual forma parte. Este estudio nos permitioì detectar las caracteriìsticas concretas y las tareas histoìricas que la Realidad Popular Chilena presenta y los desafiìos que para el Trabajo Social significa. (QUIROZ, 1972, p. 36).

El Objeto del Trabajo social, enfatiza su carácter histórico "como una construccioìn teoìrica y sistemaìtica que surgiría del anailisis del contexto global y más específicamente de contradicciones concretas y reales que presenta la sociedad en un momento histoìrico determinado" (Proyecto escuela, 1971, p.34). El objeto es la Acción Social (Praxis), de un sujeto histoìrico, protagoìnico de la transformacioìn de la realidad chilena, que se encuentra "oprimido y dominado, impedido de realizar una praxis transformadora, tanto del mundo objetivo como del subjetivo, por no poseer los medios de produccioìn y no participar real y activamente en la gestioìn poliitica, social y cultural de la sociedad". (QUIROZ, 1972, p. 36).

El Quehacer profesional será la participación comprometida "en la accioìn social del hombre dominado, mediante un proceso de Educacioìn Social, que posibilite la praxis transformadora con los aportes de la ciencia y de la teìcnica" (QUIROZ, 1972, p. 36). Ello significaba en la formación, conocer concretamente a este hombre, a través de las "prácticas productivas", 


\section{Revistg all paUtg}

\} DISPUTAS EN LA CONSTRUCCIÓN DEL TRABAJO SOCIAL - MARTÍNEZ, L. A. \}

DOI: $10.12957 /$ REP.2017.32721

en que los estudiantes se insertaban en fábricas, poblaciones, zonas rurales, y realizaban actividades concretas de los actores, que les permitiera realizar un estudio de la realidad, pasando "de una praìctica social espontaìnea a una praxis cientiìfica" posibilitando una accioìn transformadora, "cada vez maìs dinaìmica y eficiente" (QUIROZ, 1972, p.36).

Faleiros (2007), destaca el proyecto de la Escuela de la UCV, por la articulación entre la teoría y la práctica, bajo un enfoque de totalidad, histórico y crítico (p. 61); cuestionando el tecnicismo que derivaba hacia un énfasis positivista, que eludía las condiciones concretas de la realidad nacional. La crítica "al positivismo y a la focalización en problemas aislados, por un lado y, la asunción del materialismo histórico, por otro" (p. 61), plantea una "ruptura epistemológica y metodológica con el positivismo y con una visión vulgar del llamado pasaje del conocimiento sensible al conocimiento racional" (p. 61). La estructura y el proceso metodológico del Proyecto "considera la experiencia directa, el discurso, el lenguaje, la sistematización de la acción en distintos momentos a través de las aproximaciones sucesivas" (QUIROZ, 1972, p. 38), dando importancia al trabajo de teorización a través de la práctica, que permite una teoría crítica, donde "una coherencia dialéctica interna se pone en permanente comunicación con la práctica y la historia" (PROYECTO ESCUELA, 1972, p. 49). En la docencia se destaca el Seminario Taller, y "la constitución de equipos interdisciplinarios de docentes, alumnos y trabajadores que reflexionan y actúan en la realidad" (QUIROZ, 1972, p. 39), articulando tareas de investigación, docencia y acción profesional, que constituyen las bases fundamentales sobre las que se desarrolla el currículum.

El Objetivo del Trabajo Social será la praxis de la liberacioìn de los grupos dominados. "Proceso de liberacioin que se plantea en cada hombre, junto a otros en el contexto de una liberacioìn social, de acuerdo al proyecto histoìrico de estos grupos" (QUIROZ, 1971, p. 37). Dicha liberación "supone la desaparición del opresor y también de la imagen del opresor en el oprimido" (PROYECTO ESCUELA, 1972), praxis que exige Concientización (recurriendo a conceptos de Paulo Freire); Participación; Organización popular; Gestión popular; Movilización popular; y Politización. Los Campos de acción del Trabajo social tendrá dos énfasis: la Acción directa y la Educación social. La "acción directa con los sectores [...] protagonistas en el proceso de cambios estructurales. [...] trabajadores urbanos y los trabajadores rurales. La segunda perspectiva considera la concreción de nuevos valores en el contexto de una revolucioìn cultural. Tarea que consistiriìa en la educacioìn social del nuevo hombre crítico, solidario y cogestor de la estructura social" (QUIROZ, 1972, p. 37). La importancia dada a los trabajadores, no es ajena al proceso político de la UP y el efecto que tiene el ingreso de trabajadores a la universidad, quienes inicialmente estudiarán en cursos vespertinos, paralelos a los cursos regulares. El año 1970 se decide integrar ambos cursos, y para congeniar los horarios de los obreros, se opta 
el año 1971 por realizar todas las clases en horario vespertino. Este encuentro entre clases, no exento de tensiones, permitió reforzar y concretar el Proyecto de la Escuela.

dejaron hacer. Creyeron que no iba a funcionar, que iban a fracasar. Cuando vieron que no hubo deserción nada más que los estudiantes regulares y los trabajadores..., se quedaron shockeados. [...] El proyecto no estaba avalado cien por ciento por la universidad pero fue tanta la presión y el producto, cuando la escuela de Trabajo social le mostró el producto, que el noventa y nueve coma nueve por ciento había avanzado un año más, ahí se convencieron. [...] Y se dieron cuenta con la Católica también. El setenta y dos, restringieron, por qué, porque ya estábamos más comprometidos con el proceso, el proyecto político de la escuela estaba funcionando. (ELÍAS CISTERNAS, Ex alumno trabajador Escuela TSL UCV 1969-1973, entrevista 31 de agosto 2016).

El compromiso con actores populares y el empuje político, teórico y metodológico, será significativo en la concreción del proyecto de la Escuela en un contexto convulso en el interior de la universidad y el país. La quema de documentos y expedientes de alumnos, por sectores de ultraderecha, previo y posterior al golpe de estado, el 11 de septiembre de 1973, impidieron la titulación de varios estudiantes de la Escuela de la Universidad Católica de Valparaíso. Con la dictadura, la persecución será intensa. Alfredo García Vega, ex alumno y docente, será detenido el 18 de enero de 1975 junto a otros estudiantes y profesionales, y hasta hoy no se conoce su paradero. A él y a ellos dedicamos esta investigación.

\section{Conclusiones}

El proceso vivido en la Escuela de Trabajo social en Valparaíso, expresa su articulación con la recepción y reflexión, a partir de ciertas matrices teóricas, que estarán en una relación recíproca, pero no equivalente, con los procesos seguidos en otras Escuelas chilenas, donde el componente político, así como del orden del imaginario construido respecto del cambio (revolucionario, de transición, reformador) estará presente dentro de las disputas respecto de los cambios en curso, en la política, la economía y la ideología.

La incidencia de la política, en el proceso de la Escuela de Trabajo social de la Universidad Católica de Valparaíso, se distingue por la capacidad de articulación y despliegue de una diversidad de estrategias dirigidas a sensibilizar a los actores de la comunidad universitaria, para lograr la aprobación del Plan de estudios para la refundación de la Escuela bajo un nuevo planteamiento, en el Senado académico de la Universidad. La discusión sobre perspectivas teóricas, y la capacidad como escuela de debatir en una comunidad más amplia, los nuevos sentidos de la formación profesional, 


\section{ReVistg all paltg}

\} DISPUTAS EN LA CONSTRUCCIÓN DEL TRABAJO SOCIAL - MARTÍNEZ, L. A. \}

DOI: $10.12957 /$ REP.2017.32721

destacará dentro del proceso, logrando que el Proyecto se plantee explícitamente "la ruptura con lo tradicional" (FALEIROS, 2007), haciendo apropiación del concepto de historicidad, reconociendo el Trabajo social fundado en la sociedad de clases (p. 61) y en las relaciones históricas de la clase trabajadora (p. 60). La Escuela fue capaz de llevar adelante los procesos de cambio de la formación, en un país que accedía por la vía democrática, bajo una singular apropiación del socialismo. Reivindicado como un "socialismo a la chilena", por Salvador Allende, reflejará las particularidades del proceso político y cultural. Esta imagen irá cambiando y tomando forma en el espacio político, reconociendo el Trabajo social en el campo del conflicto y la disputa política para irrumpir, abrir paso, o contener un nuevo orden, aportando a la reflexión sobre el Trabajo social hasta hoy. 


\section{Referencias}

ALAYÓN, N. El Movimiento de Reconceptualización: una mirada crítica. ALAYÓN, N. (Org.). Trabajo social latinoamericano. A 40 años de la Reconceptualización. Buenos Aires: Espacio Editorial, 2007.

ALIANZA POR EL PROGRESO. Documentos básicos, 1962. Disponible en <http:/ /www.memoriachilena.cl/602/w3-article-94594.html >. Acceso en: 13 jul. 2017.

ALLARD, S. 50 años después. Inicio en Chile de la reforma universitaria de 1967. Cronología del movimiento de reforma de la Universidad Católica de Valparaíso. Valparaíso: Ediciones universitarias de Valparaíso-PUCV, 2017.

CASTORIADIS, C. Sujeto y verdad en el mundo histórico-social. Seminarios 1986-1987: la creación humana I. Argentina: Fondo de Cultura Económica, 2004.

. L'institution imaginaire de la société. Paris: Seuil, 1975.

CISTERNAS, E. Entrevista de Investigación, 31 de agosto 2016.

COUTINHO, C. N. Pluralismo: dimensiones teóricas y políticas. Cadernos Abees, São Paulo, n. 4, 1991.

DE LA VEGA, B. La situación de América Latina y el trabajo social. Revista Acción Crítica, Lima, año 1, 1976.

ESCUELA DE SERVICIO SOCIAL UNIVERSIDAD CATÓLICA DE VALPARAÍSO. Ponencia 1, Primera Convención de la Escuela de Servicio Social: Proyecto de constitución básica de la Escuela de Servicio social de la Universidad Católica de Valparaíso. Valparaíso: Universidad Católica de Valparaíso, 1968a.

. Ponencia 2, Primera Convención de la Escuela de Servicio Social: Proyecto de estructura básica de la Escuela de Servicio social UCV. Valparaíso: Universidad Católica de Valparaíso, 1968b.

FALEIROS, V. Reconceptualización del trabajo social en Brasil: ¿Una cuestión en movimiento? In: ALAYÓN, N. Trabajo social latinoamericano. A cuarenta años de la reconceptualización. Buenos Aires: Espacio Editorial, 2007.

HERNÁNDEZ, J.; RUZ, O. La reconceptualización en Chile. In: ALAYÓN, N. (Org.). Trabajo social latinoamericano. A 40 años de la Reconceptualización. Buenos Aires: Espacio Editorial, 2007.

IAMAMOTO, M. El Servicio Social en la contemporaneidad. Trabajo y formación profesional. Sao Paulo: Editorial Cortez, 2003.

. Servicio Social y división del trabajo: un análisis crítico de sus fundamentos. São Paulo: Cortez, 2001.

KISNERMAN, N. A cuarenta años de la Reconceptualización. In: ALAYÓN, N. (Org.). Trabajo social latinoamericano. A 40 años de la Reconceptualización. Buenos Aires: Espacio Editorial, 2007. 


\section{ReVistg all paltg}

\} DISPUTAS EN LA CONSTRUCCIÓN DEL TRABAJO SOCIAL - MARTÍNEZ, L. A. \}

DOI: $10.12957 /$ REP.2017.32721

KISNERMAN, N. El trabajo social en América Latina. La reconceptualización. Pensar el trabajo social. Buenos Aires: Lumen-Humanitas, 1998.

KUCZYNSKI, P. P. Explicando el contexto. In: KUCZYNSKI, P. P.; WILLIAM$\mathrm{SON}$, J. (Ed.). Después del Consenso de Washington. Relanzando el crecimiento y las reformas en América Latina. Lima: Universidad Peruana de Ciencias Aplicadas, 2003.

MARTÍNEZ, R. R.; REYES, G. E. El consenso de Washington: la instauración de las políticas neoliberales en América Latina. Revista Política y Cultura, n. 37, 2012. MARX, K., ENGELS, F. La sagrada familia. México: Grijalbo, 1967.

. Obras escogidas. Tomo III. Moscú: Editorial Progreso, 1974.

- La ideología alemana. Madrid: Akal, 2014.

NETTO, J. P. La crítica conservadora a la Reconceptualización. Acción Crítica, Lima, n. 9, 1981.

- Ditadura e Serviço Social. Uma análise do Serviço Social no Brasil pós-64. São Paulo: Cortez, 1998.

- La reconceptualización continúa viva, cuarenta años después. In: ALAYÓN, N. (Org.). Trabajo social latinoamericano. A cuarenta años de la reconceptualización. Buenos Aires: Espacio Editorial, 2007

PALMA, D. Análisis de documentos de trabajo social latinoamericano. Investigación/1, Lima, documento preliminar, 1974 (mimeo).

. Entrevista de Investigación, 30 de septiembre 2016.

PIZARRO, E. (Ed.) ¿Qué es trabajo social? Proyecto Escuela de Trabajo social UCV. Ensayos de Trabajo social, Valparaíso, n. 1, 1972.

QUIROZ, T. La nueva Escuela de trabajo social en Valparaíso. Disponible en: <https://repositorio.uc.cl/bitstream/handle/11534/6328/000381383.pdf? sequence $=1$ \&isAllowed $=y>$. Acceso en: 13 jul. 2017.

SANTOS, M. Espaço e método. São Paulo: Nobel, 1985.

YAZBEK, M. C. Fundamentos históricos e teórico-metodológicos do Serviço Social. In: CFESS, ABEPSS. Serviço Social: direitos sociais e competências profissionais. Brasília, 2009. Disponible en: <http://www.abepss.org.br/arq uivos/anexos/yazbek-201608060401395873620.pdf>. Acceso en: 4 jul. 2017.

DOI: 10.12957/rep.2017.32721

Recebido em 17 de setembro de 2017.

Aprovado para publicação em 08 de novembro de 2017.

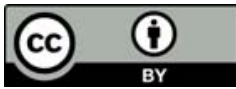

A Revista Em Pauta: Teoria Social e Realidade Contemporânea está licenciada com uma Licença Creative Commons Atribuição 4.0 Internacional. 